\title{
Building ER-PM Contacts: Keeping Calm and Ready on Alarm
}

Christopher J. Stefan

\section{Address}

MRC Laboratory for Molecular Cell Biology

University College London

Gower Street

London

WC1E 6BT

Correspondence: Christopher J. Stefan, c.stefan@ucl.ac.uk

Short title: ER-PM Contacts: Principals of Membrane Organization

http://dx.doi.org/10.1016/j.ceb.2018.03.008 


\begin{abstract}
It is well over half a century since contacts between organelles such as the endoplasmic reticulum (ER), mitochondria, and the plasma membrane (PM) were first observed in electron microscopy studies. Still, these early images of seemingly rare organelle interactions continue to capture the attention and curiosity of cell biologists even today. From seminal studies first proposing roles for organelle cross talk in excitable cells, the field has now expanded to cover nearly all aspects of eukaryotic cell biology, from calcium and membrane lipid transport to vesicular trafficking, cell signaling, metabolism, and homeostasis. This review highlights recent discoveries pointing to vital roles for ER-PM contacts in membrane lipid dynamics and organization.
\end{abstract}




\section{Introduction}

Regions of the peripheral endoplasmic reticulum (ER) closely apposed to the plasma membrane (PM) were revealed in elegant detail by electron microscopy (EM) studies on muscle cells [1], where they play important roles in calcium $\left(\mathrm{Ca}^{2+}\right)$-mediated excitation-contraction events [2]. A few years later, Jack Rosenbluth reported "subsurface cisterns" in neurons [3], which are now recognized as ER-PM contacts and have been described in greater morphological detail with recent advances in cryo-EM tomography $[4,5]$. At the time of their discovery the role of "subsurface cisterns" were obscure, but because these structures were prominent in neurons and muscle cells Rosenbluth surmised that they might be integral to metabolic 'peculiarities', including ion transport and rapid membrane lipid dynamics, occurring in excitable cells. This prediction has turned out to be the case, and ER-PM contacts are now widely accepted as important sites for non-vesicular $\mathrm{Ca}^{2+}$ and membrane lipid transport. Moreover, it is now evident that ER-PM contacts are ubiquitous structures that function not only in excitable cells but also numerous cell types across single- and multi-cellular eukaryotic organisms.

Conserved roles for ER-PM contacts in membrane lipid dynamics and homeostasis have been elucidated by the identification of proteins that form and function at these important cellular structures. In particular, the identification of lipid transfer proteins that function at these sites has greatly aided our understanding of how ER-PM contacts control membrane lipid composition and organization. Within the past few 
years, we have learned a great deal more about the selective transport of lipids between the ER and PM. Cells have a vast toolkit of lipid transfer proteins on hand for the discrete movement of lipids, for anterograde transport to the PM versus retrograde transport to the ER, and for the establishment of lipid concentration gradients versus lipid equilibration. Importantly, we are now learning more about the regulatory mechanisms that control the directional movements of lipids at ER-PM contacts as needed. As such, a key concept is emerging in the field. Namely, distinct ER-PM contacts with unique features and functions are built on demand, in response to intrinsic and extrinsic physiological cues, to balance membrane lipid homeostasis and dynamics as needed.

\section{Keeping Calm: ER-PM Contacts in Membrane Organization and}

\section{Homeostasis}

Membrane integrity ensures cellular integrity. As such, membrane status is continuously assessed and maintained through careful control of membrane protein and lipid composition. Lipid molecules have distinct physical properties that determine their organization in a cellular membrane bilayer. Accordingly, the PM has a unique membrane lipid composition that determines its mechano-physical properties, organization, and identity from other membrane compartments in the cell [6]. For example, the PM is enriched in sterol, sphingolipids, and certain phospholipids as compared to other cellular membranes (Figure 1) $[7,8]$. In particular, high levels of the phospholipid phosphatidylserine maintain an overall negative charge at the cytosolic face of the PM, a key feature of the PM [9]. The PM displays 
unique features at the lipid species level as well, represented in distinctive acyl chain properties. First, the PM is enriched in long acyl chain sphingo- and phospholipid species in order to suit the length of PM transmembrane domain proteins $[7,8,10]$. Moreover, the PM is enriched in lipids with saturated acyl chains thought to preferentially associate in liquid-ordered domains stabilized by sterol lipids $[7,8]$. In contrast, the ER membrane is less ordered, more fluid, thinner, and less charged than the PM. The chemical and physical properties of the ER membrane are defined by low sterol, sphingolipid, and phosphatidylserine content and, in converse, higher levels of neutral aminophospholipids with shorter, unsaturated acyl chain species (Figure 1).

The conserved PM and ER lipid identity codes are created by the selective delivery of lipids between the ER and PM by specific lipid transfer proteins and possibly by vesicular trafficking (Figure 1), as spontaneous lipid transfer between membranes is energetically unfavorable and potentially non-specific [11]. In particular, members of a conserved family of lipid transfer proteins, the oxysterol-binding protein related proteins (ORP), are thought to be crucial for the establishment and maintenance of PM lipid identity (Figure 2). ORP family members transfer newly synthesized cholesterol and phosphatidylserine from the ER in exchange for phosphatidylinositol 4-phosphate (PI4P) at the PM [12-15]. In this manner, cells utilize PI4P metabolism for directional transport of lipids from the ER to the PM (i.e. transport from the ER to the PM against a concentration gradient). While exchange of one lipid molecule for 
another lipid molecule may not result in net lipid gain, this mechanism effectively results in the enrichment of sterol and phosphatidylserine at the PM.

ER-PM contacts may then have essential roles in PM organization and homeostasis (keeping calm). In support of this notion, loss of proteins previously shown to form and function at ER-PM contacts in yeast, namely Scs2/22 (VAP orthologs), the Tcb1/2/3 tricalbin proteins (Extended synaptotagmin orthologs, E-Syt proteins), and Ist2 (a TMEM16 family member), results in loss of PM integrity upon membrane stress conditions [16]. Upon physiological membrane stress conditions, phosphoinositide kinase signaling at the PM triggers TORC2-induced sphingolipid synthesis in the ER necessary to maintain cellular integrity. Defects in ER-PM contact formation results in mis-regulation of phosphoinositide lipid metabolism, $\mathrm{Ca}^{2+}$ signaling, sphingolipid synthesis, and ultimately loss of cellular integrity [16]. Another cellular stress response pathway in yeast, the Rim101-ESCRT coupled membrane sensing and sculpting pathway, is activated upon disruptions in ER-PM contacts further suggesting important roles for these structures in the control of membrane organization [17].

Membrane homeostasis requires both the selective delivery and removal of lipids to adjust membrane lipid composition as needed. In particular, sterol lipids are key determinants of membrane bilayer packing order (i.e. liquid ordered and disordered domains), fluidity, and integrity [6]. As such, their levels and distribution within a 
membrane bilayer must be tightly regulated. Conserved ER-localized StART-like (StAR-related lipid-transfer) domain proteins, named Lam/Ltc/Ysp proteins in yeast and collectively referred to as the StARkin domain family, are suggested to direct retrograde transfer of sterol lipids from the PM to the ER [18-22]. This activity may oppose ORP-mediated sterol transport to the PM. In line with this, ORP and StARkin transport activities occur at spatially distinct ER-PM contacts (Figure 2) [19]. This implies that specific sterol transfer systems are built and coordinated as needed and that the balance of sterol delivery and removal ensures PM homeostasis. Consistent with this, loss of StARkin proteins at ER-PM contacts in yeast results in TORC2mediated stress responses potentially due to perturbations in PM sterol organization [23].

Specialized ER-PM contacts are also formed in response to changes in ER status. A well-studied example is store-operated calcium entry (SOCE) whereby the ERlocalized STIM proteins bind and activate the PM-localized Orai1 channel to maintain $\mathrm{ER} \mathrm{Ca}^{2+}$ homeostasis [2]. The unfolded protein response (UPR) sensor PERK is recently implicated in this process [24]. Upon depletion of ER $\mathrm{Ca}^{2+}$ stores and ER stress, PERK signaling drives actin network reorganization to assist STIM protein ERPM targeting and SOCE. As PERK is also activated by ER membrane perturbations, particularly the accumulation of 'PM-like' saturated acyl chain lipid species [25], this stress response pathway may modulate ER and PM lipid homeostasis as well. While this remains speculative, PERK facilitates targeting of the lipid transfer protein E-Syt1 to ER-PM contacts [24] (see below for further discussion of the E-Syt proteins). 
Taken together, recent studies establish that ER-PM contacts are built as needed to control PM lipid composition, ensuring membrane homeostasis and integrity.

\section{Ready on Alarm: Inducible ER-PM Contacts in Regulated Exocytosis and Endocytosis}

In addition to membrane organization, ER-PM contacts support dynamic vesicular trafficking events taking place at the PM [2]. Phosphoinositide lipid and $\mathrm{Ca}^{2+}$ dynamics regulated at ER-PM contacts are vital for modulatory neurotransmitter release, pulsatile insulin secretion from pancreatic $\beta$ cells, and regulated exocytosis in immune cells. It is generally understood that receptor-mediated phospholipase C (PLC) activation results in phosphatidylinositol 4,5 bis-phosphate hydrolysis, $\mathrm{Pl}(4,5) \mathrm{P}_{2}$, generating second messenger molecules including diacylglycerol and $\mathrm{Ca}^{2+}$ signals that trigger exocytic events (Figure 3) [26]. However, we are learning more about how $\mathrm{PI}(4,5) \mathrm{P}_{2}$ hydrolysis, even upon slight physiological reductions in cellular pools [27], sets forth a chain of lipid transfer and metabolic reactions needed to sustain $\mathrm{PI}(4,5) \mathrm{P}_{2}$ pools for multiple rounds of exocytosis in response to extracellular stimuli $[28,29]$. These events are mediated by inducible, regulated lipid transfer protein activities that are activated in response (ready on alarm) to the same physiological stimuli that trigger exocytosis (e.g. elevated glucose in the case of $\beta$ cells, and antigen stimulation in mast cells). 
A striking and yet underappreciated observation is that levels of PI4P, a precursor to $\mathrm{PI}(4,5) \mathrm{P}_{2}$, rise simultaneously with $\mathrm{PI}(4,5) \mathrm{P}_{2}$ hydrolysis at the PM (Figure 3) $[30,31]$. This implies that robust systems for $\mathrm{PI}(4,5) \mathrm{P}_{2}$ re-synthesis are engaged upon PLC activation. Rapid recycling of diacylglycerol, a direct product of PLC-mediated $\mathrm{PI}(4,5) \mathrm{P}_{2}$ hydrolysis, facilitates efficient $\mathrm{PI}(4,5) \mathrm{P}_{2}$ re-synthesis in a process known as the phosphoinositide cycle [26]. Because diacylglycerol is generated from $\mathrm{PI}(4,5) \mathrm{P}_{2}$ at the PM and phosphatidylinositol, the precursor to $\mathrm{PI} 4 \mathrm{P}$ and $\mathrm{PI}(4,5) \mathrm{P}_{2}$ is synthesized by the CDP-diacylglycerol pathway in the ER, lipid transfer protein activities at ER-PM contacts are instrumental during the phosphoinositide cycle. Recent studies on the Nir2 phosphatidylinositol transfer protein and its ortholog RdgB in Drosophila have provided mechanistic insight into this process [32-36].

Diacylglycerol produced from $\mathrm{PI}(4,5) \mathrm{P}_{2}$ hydrolysis is first converted to phosphatidic acid by diacylglycerol kinase at the PM. Nir2 then translocates to the PM by binding phosphatidic acid. At ER-PM contacts, Nir2 transfers phosphatidic acid to the ER for phosphatidylinositol synthesis (via the CDP-diacylglycerol pathway) and in turn transports phosphatidylinositol back to the $\mathrm{PM}$ for $\mathrm{PI} 4 \mathrm{P}$ and $\mathrm{PI}(4,5) \mathrm{P}_{2}$ synthesis. Interestingly, targeting of Nir2 to ER-PM contacts is further specified by binding the ER-localized VAP proteins that also recruit certain ORP isoforms [35]. Thus in response to stimuli that trigger PLC-regulated exocytosis, ER-PM contacts may switch from sites of PI4P exchange (e.g. PI4P and sterol, Figure 2) to sites that promote $\mathrm{PI} 4 \mathrm{P}$ and $\mathrm{PI}(4,5) \mathrm{P}_{2}$ synthesis (Figure 3). 
Additional lipid transfer proteins function at ER-PM contacts in response to PLCgenerated signals. The extended synaptotagmin (E-Syt) proteins are $\mathrm{Ca}^{2+}$-activated ER-PM tether proteins that transfer PLC-generated diacylglycerol from the PM to the ER [37], potentially working in concert with the Nir2 protein during the phosphoinositide cycle [32]. E-Syts utilize their SMP domain (synaptotagmin-like mitochondrial lipid-binding protein domain) to transfer lipids [37-39], but while this activity is apparently selective for diacylglycerol in vivo [37], the E-Syts are not selective in vitro. Both biochemical and structural studies demonstrate that SMP domains bind various glycerolipids and phospholipids and transfer them down a concentration gradient (from high to low) [37-39]. This implies that E-Syt activity must be tightly regulated and only allowed to proceed in response to specific stimuli. Otherwise E-Syt proteins may non-selectively transfer phospholipids enriched at the PM, including phosphatidylserine, back to the ER. This would potentially undue PI4Pand ORP-mediated phosphatidylserine transfer to the PM, a critical determinant for PM identity and function. Consistent with this, cells lacking E-Syt proteins do not display changes in major glycerolipid species in the absence of stimuli that trigger PLC signaling [37].

Accordingly, both in vitro and in vivo studies have uncovered important E-Syt regulatory mechanisms. The crystal structure of the SMP domain from E-Syt2 revealed a $\beta$-barrel structure similar to other tubular lipid-binding (TULIP) domain proteins [38]. Interestingly, the SMP domain dimerizes forming a cylindrical channel lined with hydrophobic residues that can accommodate glycerophospholipids. 
However, the SMP dimer spans approximately $9 \mathrm{~nm}$ in length, suggesting that E-Syt lipid transfer activity may only occur at sites where the ER and PM are closely tethered and may not take place at ORP-mediated ER-PM contacts typically 25-30 $\mathrm{nm}$ apart (Figure 2). Indeed, SMP domain-mediated lipid transfer in vitro requires tight membrane tethering by $\mathrm{Ca}^{2+}$-bound $\mathrm{C} 2$ domains in the E-Syt proteins $[37,39]$. Notably, E-Syt1 undergoes autoinhibition and $\mathrm{Ca}^{2+}$ binding relieves autoinhibitory interactions between the $\mathrm{C} 2 \mathrm{~A}$ and SMP domains as well as between $\mathrm{C} 2 \mathrm{C}$ and $\mathrm{C} 2 \mathrm{E}$ that prevent binding to $\mathrm{PI}(4,5) \mathrm{P}_{2}$ in the $\mathrm{PM}[40]$. In support of the in vitro evidence, in vivo studies show that $\mathrm{Ca}^{2+}$ influx by the SOCE pathway and voltage gated $\mathrm{Ca}^{2+}$ channels triggers E-Syt1 recruitment to ER-PM contacts [41]. Moreover, $\mathrm{Ca}^{2+}$ influx shortens the distance between the ER and PM at E-Syt1-dependent contact sites, as revealed by cryo-EM tomography [4]. Thus, E-Syt proteins specifically respond to external stimuli that trigger PLC signaling, $\mathrm{Ca}^{2+}$ influx, and regulated exocytosis. Importantly, this shared $\mathrm{Ca}^{2+}$ regulatory mechanism indicates vital cross talk between ER-associated PM zones and ER-free vesicular trafficking zones (Figure 3).

While ER-PM contacts are crucial sites for SOCE and $\mathrm{PI}(4,5) \mathrm{P}_{2}$ re-synthesis following PLC activation, another recent study has suggested that ER-PM contacts are important sites for PI4P homeostasis by E-Syt2 and the PI4P phosphatase Sac1 [42]. PI4P directly activates SOCE via the Orai1 channel [43] and is central to $\mathrm{PI}(4,5) \mathrm{P}_{2}$ synthesis; as such, $\mathrm{PI} 4 \mathrm{P}$ levels must be tightly regulated. In response to intense PLC activity and $\mathrm{PI}(4,5) \mathrm{P}_{2}$ hydrolysis, E-Syt2-dependent ER-PM contacts are depleted facilitating PI4P-mediated SOCE and $\mathrm{PI}(4,5) \mathrm{P}_{2}$ re-synthesis [42]. Following 
SOCE and $\mathrm{PI}(4,5) \mathrm{P}_{2}$ re-synthesis, E-Syt2 ER-PM contacts are reformed allowing Sac1-mediated PI4P regulation preventing excessive $\mathrm{Ca}^{2+}$ and $\mathrm{PI}(4,5) \mathrm{P}_{2}$ overshoot [42]. Altogether, recent findings have highlighted several key roles in the homeostatic control of $\mathrm{PI}(4,5) \mathrm{P}_{2}$ and $\mathrm{Ca}^{2+}$ signaling. Disruptions in ER-PM cross talk result in impaired $\mathrm{PI}(4,5) \mathrm{P}_{2}$ synthesis $[32,33,35,36,44]$ and mis-regulation of PI4P-dependent $\mathrm{Ca}^{2+}$ signaling $[16,42,44,45]$ (Figure 3 ).

Considering the vital roles the E-Syt proteins serve in $\mathrm{PI}(4,5) \mathrm{P}_{2}$ and $\mathrm{Ca}^{2+}$ regulation, one might expect they are essential for cellular function and viability. Surprisingly, two recent studies independently report that mice lacking E-Syt proteins do not display obvious defects $[46,47]$. This may suggest that the E-Syt proteins have highly specialized roles. Another plausible explanation is additional lipid transfer proteins compensate for loss of E-Syt protein function. Interestingly, the SMP domain protein TMEM24 controls $\mathrm{Ca}^{2+}$ and $\mathrm{PI}(4,5) \mathrm{P}_{2}$ signaling necessary for regulated insulin secretion in pancreatic $\beta$ cells [48]. The TMEM24 SMP domain preferentially binds and transfers phosphatidylinositol from the ER to the PM. Upon loss of TMEM24 in $\beta$ cells, $\mathrm{PI}(4,5) \mathrm{P}_{2}$ synthesis is impaired decreasing the generation of $\mathrm{Ca}^{2+}$ signals that trigger insulin release. It remains unknown whether TMEM24 controls phosphoinositide and $\mathrm{Ca}^{2+}$ signaling in other cell types. Moreover, additional members of the SMP/TULIP superfamily exist and their potential roles at ER-PM contacts remain to be explored $[49,50]$. 
Previous studies implicate $\mathrm{Ca}^{2+}, \mathrm{PI}(4,5) \mathrm{P}_{2}$, and actin oscillations during regulated exocytosis [51,52]. Given the important roles the Nir2, E-Syt, and TMEM24 proteins serve in $\mathrm{PI}(4,5) \mathrm{P}_{2}$ and $\mathrm{Ca}^{2+}$ regulation, ER-PM contacts may be generally involved in vesicular trafficking events at the PM. In support of this, the majority of exocytic and clathrin-mediated endocytic events in HEK293 cells appear to occur in close proximity to ER-PM contacts [53]. Another recent study suggests that ER-PM contacts promote actin polymerization during late stages of clathrin-mediated endocytic internalization in yeast [54]. Intriguingly, ER-PM contacts are also implicated in clathrin-independent epidermal growth factor receptor internalization [55], likely through modulation of phosphoinositide and $\mathrm{Ca}^{2+}$ signaling. Thus while ER-PM contacts and ER-free PM zones may appear to be spatially distinct and have different physiological roles (non-vesicular transport versus vesicle trafficking), it is becoming increasingly clear that these PM domains are functionally interconnected (Figure 3).

\section{Summary}

ER-PM contacts are important sites for membrane lipid homeostasis and organization. Because PM composition is continuously adjusted according to physiological cues, distinct ER-PM contacts are assembled and disassembled as needed. This is accomplished by the specific recruitment and regulation of lipid transfer proteins that function at these sites. Consequently, differential activities at ER-PM contacts can effectively influence sterol and phospholipid distribution, phosphoinositide metabolism, and vesicle trafficking events. We must continue to 
examine the interplay between lipid transfer proteins and how they may act in concert for directional net movements of lipids between the ER and PM. As such, future studies on ER-PM contacts will have tremendous impact on our understanding of cellular architecture and physiology.

\section{Acknowledgements}

I thank members of the Stefan laboratory, especially Dr. Taki Nishimura, for helpful discussions. The Stefan laboratory is supported by MRC funding to the MRC LMCB University Unit at UCL, award code MC_UU_12018/6.

\section{Competing interests}

The author declares no conflicts of interest. 


\section{References and recommended reading}

Papers of particular interest, published within the period of review, have been highlighted as:

- of special interest

•• of outstanding interest

1. Porter KR, Palade GE: Studies on the endoplasmic reticulum. III. Its form and distribution in striated muscle cells. J Biophys Biochem Cytol 1957, 3:269300 .

2. Stefan CJ, Manford AG, Emr SD: ER-PM connections: sites of information transfer and inter-organelle communication. Curr Opin Cell Biol 2013, 25:434-442.

3. Rosenbluth J: Subsurface cisterns and their relationship to the neuronal plasma membrane. J Cell Biol 1962, 13:405-421.

4. Fernandez-Busnadiego R, Saheki Y, De Camilli P: Three-dimensional architecture of extended synaptotagmin-mediated endoplasmic reticulum-plasma membrane contact sites. Proc Natl Acad Sci U S A 2015, 112:E2004-2013.

5. Wu Y, Whiteus C, Xu CS, Hayworth KJ, Weinberg RJ, Hess HF, De Camilli P: Contacts between the endoplasmic reticulum and other membranes in neurons. Proc Natl Acad Sci U S A 2017, 114:E4859-E4867.

6. Holthuis JC, Menon AK: Lipid landscapes and pipelines in membrane homeostasis. Nature 2014, 510:48-57.

7. Surma MA, Klose C, Klemm RW, Ejsing CS, Simons K: Generic sorting of raft lipids into secretory vesicles in yeast. Traffic 2011, 12:1139-1147.

8. Schneiter R, Brugger B, Sandhoff R, Zellnig G, Leber A, Lampl M, Athenstaedt K, Hrastnik C, Eder S, Daum G, et al.: Electrospray ionization tandem mass spectrometry (ESI-MS/MS) analysis of the lipid molecular species composition of yeast subcellular membranes reveals acyl chain-based sorting/remodeling of distinct molecular species en route to the plasma membrane. J Cell Biol 1999, 146:741-754.

9. Yeung T, Heit B, Dubuisson JF, Fairn GD, Chiu B, Inman R, Kapus A, Swanson M, Grinstein S: Contribution of phosphatidylserine to membrane surface charge and protein targeting during phagosome maturation. $J$ Cell Biol 2009, 185:917-928. 
10. Borgese N: Getting membrane proteins on and off the shuttle bus between the endoplasmic reticulum and the Golgi complex. J Cell Sci 2011, 129:1537-1545.

11. Merklinger E, Schloetel JG, Spitta L, Thiele C, Lang T: No Evidence for Spontaneous Lipid Transfer at ER-PM Membrane Contact Sites. J Membr Biol 2016, 249:41-56.

12. Chung J, Torta F, Masai K, Lucast L, Czapla H, Tanner LB, Narayanaswamy P, Wenk MR, Nakatsu F, De Camilli P: INTRACELLULAR TRANSPORT. PI4P/phosphatidylserine countertransport at ORP5- and ORP8-mediated ER-plasma membrane contacts. Science 2015, 349:428-432.

13. de Saint-Jean M, Delfosse V, Douguet D, Chicanne G, Payrastre B, Bourguet W, Antonny B, Drin G: Osh4p exchanges sterols for phosphatidylinositol 4phosphate between lipid bilayers. J Cell Biol 2011, 195:965-978.

14. Moser von Filseck J, Copic A, Delfosse V, Vanni S, Jackson CL, Bourguet W, Drin G: INTRACELLULAR TRANSPORT. Phosphatidylserine transport by ORP/Osh proteins is driven by phosphatidylinositol 4-phosphate. Science 2015, 349:432-436.

15. Sohn M, Ivanova P, Brown HA, Toth DJ, Varnai P, Kim YJ, Balla T: LenzMajewski mutations in PTDSS1 affect phosphatidylinositol 4-phosphate metabolism at ER-PM and ER-Golgi junctions. Proc Natl Acad Sci U S A 2016, 113:4314-4319.

-The study by Sohn et al., along with studies by Chung et al. and Moser von Filseck et al. [12,14], provide important mechanistic insight into ORP-mediated phosphatidlylserine transport to the PM. The Sohn et al. study demonstrates that lipid metabolism in the ER is tightly coordinated with phosphoinositide metabolism at the PM. This regulation is critical for human physiology, as disruptions in phosphatidlylserine metabolism and transport are implicated in Lenz-Majewski syndrome.

16. Omnus DJ, Manford AG, Bader JM, Emr SD, Stefan CJ: Phosphoinositide kinase signaling controls ER-PM cross-talk. Mol Biol Cell 2016, 27:11701180.

17. Obara K, Kihara A: The Rim101 pathway contributes to ER stress adaptation through sensing the state of plasma membrane. Biochem J 2016, 474:5163.

18. Gatta AT, Sauerwein AC, Zhuravleva A, Levine TP, Matthews S: Structural insights into a StART-like domain in Lam4 and its interaction with sterol ligands. Biochem Biophys Res Commun 2018, 495:2270-2274. 
19. Gatta AT, Wong LH, Sere YY, Calderon-Norena DM, Cockcroft S, Menon AK, Levine TP: A new family of StART domain proteins at membrane contact sites has a role in ER-PM sterol transport. Elife 2015, 4.

20. Horenkamp FA, Valverde DP, Nunnari J, Reinisch KM: Molecular basis for sterol transport by StART-like lipid transfer domains. EMBO J 2018.

21. Jentsch JA, Kiburu I, Pandey K, Timme M, Ramlall T, Levkau B, Wu J, Eliezer D, Boudker O, Menon AK: Structural basis of sterol binding and transport by a yeast StARkin domain. J Biol Chem 2018.

22. Tong J, Manik MK, Im YJ: Structural basis of sterol recognition and nonvesicular transport by lipid transfer proteins anchored at membrane contact sites. Proc Natl Acad Sci U S A 2018, 115:E856-E865.

23. Murley A, Yamada J, Niles BJ, Toulmay A, Prinz WA, Powers T, Nunnari J: Sterol transporters at membrane contact sites regulate TORC1 and TORC2 signaling. J Cell Biol 2017, 216:2679-2689.

24. van Vliet AR, Giordano F, Gerlo S, Segura I, Van Eygen S, Molenberghs G, Rocha S, Houcine A, Derua R, Verfaillie T, et al.: The ER Stress Sensor PERK Coordinates ER-Plasma Membrane Contact Site Formation through Interaction with Filamin-A and F-Actin Remodeling. Mol Cell 2017, 65:885-899 e886.

-The study by van Vliet et al. reports an unexpected role for the ER stress response protein PERK in the formation of ER-PM contacts during store-operated $\mathrm{Ca}^{2+}$ entry (SOCE). Upon depletion of ER Ca ${ }^{2+}$ stores, the ER-localized STIM1 protein is known to translocate to ER-PM contacts where it activates SOCE through interactions with the Orai1 $\mathrm{Ca}^{2+}$ channel at the PM. The current study shows that ER $\mathrm{Ca}^{2+}$ release triggers PERK signalling that in turn controls actin cytoskeletal dynamics necessary for STIM1 recruitment to ER-PM contacts and SOCE. In addition to STIM1, PERK regulates E-Syt1 targeting to ER-PM contacts possibly to direct membrane lipid transport in response to ER stress.

25. Volmer R, van der Ploeg K, Ron D: Membrane lipid saturation activates endoplasmic reticulum unfolded protein response transducers through their transmembrane domains. Proc Natl Acad Sci U S A 2013, 110:46284633.

26. Michell RH: Inositol phospholipids and cell surface receptor function. Biochim Biophys Acta 1975, 415:81-47. 
27. Dickson EJ, Falkenburger BH, Hille B: Quantitative properties and receptor reserve of the $\mathrm{IP}(3)$ and calcium branch of $\mathrm{G}(\mathrm{q})$-coupled receptor signaling. J Gen Physiol 2013, 141:521-535.

28. Chang CL, Liou J: Homeostatic regulation of the PI(4,5)P2-Ca(2+) signaling system at ER-PM junctions. Biochim Biophys Acta 2016, 1861:862-873.

29. Cockcroft S, Raghu P: Topological organisation of the phosphatidylinositol 4,5-bisphosphate-phospholipase $C$ resynthesis cycle: PITPs bridge the ER-PM gap. Biochem J 2016, 473:4289-4310.

30. Toth JT, Gulyas G, Toth DJ, Balla A, Hammond GR, Hunyady L, Balla T, Varnai $P$ : BRET-monitoring of the dynamic changes of inositol lipid pools in living cells reveals a PKC-dependent Ptdlns4P increase upon EGF and M3 receptor activation. Biochim Biophys Acta 2016, 1861:177-187.

31. Wuttke A, Sagetorp J, Tengholm A: Distinct plasma-membrane Ptdlns(4)P and Ptdlns(4,5)P2 dynamics in secretagogue-stimulated beta-cells. J Cell Sci 2010, 123:1492-1502.

32. Chang CL, Hsieh TS, Yang TT, Rothberg KG, Azizoglu DB, Volk E, Liao JC, Liou $\mathrm{J}$ : Feedback regulation of receptor-induced $\mathrm{Ca2}+$ signaling mediated by E-Syt1 and Nir2 at endoplasmic reticulum-plasma membrane junctions. Cell Rep 2013, 5:813-825.

33. Chang CL, Liou J: Phosphatidylinositol 4,5-Bisphosphate Homeostasis Regulated by Nir2 and Nir3 Proteins at Endoplasmic Reticulum-Plasma Membrane Junctions. J Biol Chem 2015, 290:14289-14301.

34. Kim S, Kedan A, Marom M, Gavert N, Keinan O, Selitrennik M, Laufman O, Lev $S$ : The phosphatidylinositol-transfer protein Nir2 binds phosphatidic acid and positively regulates phosphoinositide signalling. EMBO Rep 2013, 14:891-899.

35. Kim YJ, Guzman-Hernandez ML, Wisniewski E, Balla T: PhosphatidylinositolPhosphatidic Acid Exchange by Nir2 at ER-PM Contact Sites Maintains Phosphoinositide Signaling Competence. Dev Cell 2015, 33:549-561.

36. Yadav S, Garner K, Georgiev P, Li M, Gomez-Espinosa E, Panda A, Mathre S, Okkenhaug H, Cockcroft S, Raghu P: RDGBalpha, a Ptdlns-PtdOH transfer protein, regulates G-protein-coupled Ptdlns(4,5)P2 signalling during Drosophila phototransduction. J Cell Sci 2015, 128:3330-3344. 
37. Saheki Y, Bian X, Schauder CM, Sawaki Y, Surma MA, Klose C, Pincet F, Reinisch KM, De Camilli P: Control of plasma membrane lipid homeostasis by the extended synaptotagmins. Nat Cell Biol 2016, 18:504-515.

-Together, the studies by Saheki et al. and Bian et al. [40•] provide new insight into the function and regulation of the SMP domain-containing E-Syt1 protein at ER-PM contacts. Saheki et al. show that E-Syt1 is a $\mathrm{Ca}^{2+}$-regulated lipid transfer protein that transfers phospholipase C-generated diacylglycerol from the PM to the ER. Bian et al. show that E-Syt1 is auto-inhibited through intra-molecular interactions between its $\mathrm{C} 2 \mathrm{~A}$ and SMP domains in the absence of stimuli that elevate cytosolic $\mathrm{Ca}^{2+}$. This inhibition is released upon $\mathrm{Ca}^{2+}$ binding to the $\mathrm{C} 2 \mathrm{~A}$ domain, allowing SMP domaindependent diacylglycerol transport. Thus, E-Syt1 may participate in phosphoinositide lipid signaling specifically in response to stimuli that trigger phospholipase Cmediated $\mathrm{PI}(4,5) \mathrm{P}_{2}$ hydrolysis.

38. Schauder CM, Wu X, Saheki Y, Narayanaswamy P, Torta F, Wenk MR, De Camilli $P$, Reinisch KM: Structure of a lipid-bound extended synaptotagmin indicates a role in lipid transfer. Nature 2014, 510:552-555.

39. Yu H, Liu Y, Gulbranson DR, Paine A, Rathore SS, Shen J: Extended synaptotagmins are $\mathrm{Ca2+-dependent} \mathrm{lipid} \mathrm{transfer} \mathrm{proteins} \mathrm{at} \mathrm{membrane}$ contact sites. Proc Natl Acad Sci U S A 2016, 113:4362-4367.

40. Bian $X$, Saheki Y, De Camilli P: $\mathbf{C a ( 2 + )}$ releases E-Syt1 autoinhibition to couple ER-plasma membrane tethering with lipid transport. EMBO J 2017.

41. Idevall-Hagren $O$, Lu A, Xie $B$, De Camilli $P$ : Triggered Ca2+ influx is required for extended synaptotagmin 1-induced ER-plasma membrane tethering. EMBO J 2015, 34:2291-2305.

42. Dickson EJ, Jensen JB, Vivas O, Kruse M, Traynor-Kaplan AE, Hille B: Dynamic formation of ER-PM junctions presents a lipid phosphatase to regulate phosphoinositides. J Cell Biol 2016, 213:33-48.

-The study by Dickson et al. describes a distinct role for E-Syt2-dependent ER-PM contacts in phosphoinositide and $\mathrm{Ca}^{2+}$ homeostasis. E-Syt2 places the phosphoinositide phosphatase Sac1 in proximity to the PM where it may regulate PI4P levels by a mechanism that is not completely understood. Interestingly, upon activation of receptors coupled to phospholipase $\mathrm{C}$ activity and $\mathrm{PI}(4,5) \mathrm{P}_{2}$ hydrolysis, E-Syt2- and Sac1-containing ER-PM contacts are disassembled resulting in increased PI4P levels for store-operated $\mathrm{Ca}^{2+}$ entry (SOCE) and $\mathrm{PI}(4,5) \mathrm{P}_{2}$ resynthesis. SOCE reforms E-Syt2-mediated ER-PM contacts to restore PI4P homeostasis and return to the resting state. 
43. Korzeniowski MK, Popovic MA, Szentpetery Z, Varnai P, Stojilkovic SS, Balla T: Dependence of STIM1/Orai1-mediated calcium entry on plasma membrane phosphoinositides. J Biol Chem 2009, 284:21027-21035.

44. Manford AG, Stefan CJ, Yuan HL, Macgurn JA, Emr SD: ER-to-plasma membrane tethering proteins regulate cell signaling and ER morphology. Dev Cell 2012, 23:1129-1140.

45. Giordano F, Saheki Y, Idevall-Hagren O, Colombo SF, Pirruccello M, Milosevic I, Gracheva EO, Bagriantsev SN, Borgese N, De Camilli P: PI(4,5)P(2)dependent and $\mathrm{Ca}(2+)$-regulated ER-PM interactions mediated by the extended synaptotagmins. Cell 2013, 153:1494-1509.

46. Sclip A, Bacaj T, Giam LR, Sudhof TC: Extended Synaptotagmin (ESyt) Triple Knock-Out Mice Are Viable and Fertile without Obvious Endoplasmic Reticulum Dysfunction. PLoS One 2016, 11:e0158295.

47. Tremblay MG, Moss T: Loss of all 3 Extended Synaptotagmins does not affect normal mouse development, viability or fertility. Cell Cycle 2016, 15:2360-2366.

48. Lees JA, Messa M, Sun EW, Wheeler H, Torta F, Wenk MR, De Camilli P, Reinisch KM: Lipid transport by TMEM24 at ER-plasma membrane contacts regulates pulsatile insulin secretion. Science 2017, 355.

- The study by Lees et al. identifies the SMP domain protein TMEM24 as an ERlocalized lipid transfer protein in pancreatic $\beta$ cells. TMEM24 serves as phosphatidylinositol transfer protein at ER-PM contacts necessary for $\mathrm{PI}(4,5) \mathrm{P}_{2}$ and $\mathrm{Ca}^{2+}$ oscillations in response to physiological stimuli. Importantly, this study demonstrates an essential role for the TMEM24 protein and membrane lipid dynamics taking place at ER-PM contacts in regulated insulin secretion, which is compromised in type II diabetes.

49. Alva V, Lupas AN: The TULIP superfamily of eukaryotic lipid-binding proteins as a mediator of lipid sensing and transport. Biochim Biophys Acta 2016, 1861:913-923.

50. Wong LH, Levine TP: Tubular lipid binding proteins (TULIPs) growing everywhere. Biochim Biophys Acta 2017, 1864:1439-1449.

51. Wollman R, Meyer T: Coordinated oscillations in cortical actin and Ca2+ correlate with cycles of vesicle secretion. Nat Cell Biol 2012, 14:12611269. 
52. Wu M, Wu X, De Camilli P: Calcium oscillations-coupled conversion of actin travelling waves to standing oscillations. Proc Natl Acad Sci U S A 2013, 110:1339-1344.

53. Fox PD, Haberkorn CJ, Weigel AV, Higgins JL, Akin EJ, Kennedy MJ, Krapf D, Tamkun MM: Plasma membrane domains enriched in cortical endoplasmic reticulum function as membrane protein trafficking hubs. Mol Biol Cell 2013, 24:2703-2713.

54. Encinar Del Dedo J, Idrissi FZ, Fernandez-Golbano IM, Garcia P, Rebollo E, Krzyzanowski MK, Grotsch H, Geli MI: ORP-Mediated ER Contact with Endocytic Sites Facilitates Actin Polymerization. Dev Cell 2017, 43:588602 e586.

55. Caldieri G, Barbieri E, Nappo G, Raimondi A, Bonora M, Conte A, Verhoef L, Confalonieri S, Malabarba MG, Bianchi F, et al.: Reticulon 3-dependent ERPM contact sites control EGFR nonclathrin endocytosis. Science 2017, 356:617-624. 


\title{
Figures and Figure Legends
}

\author{
Stefan, Figure 1
}

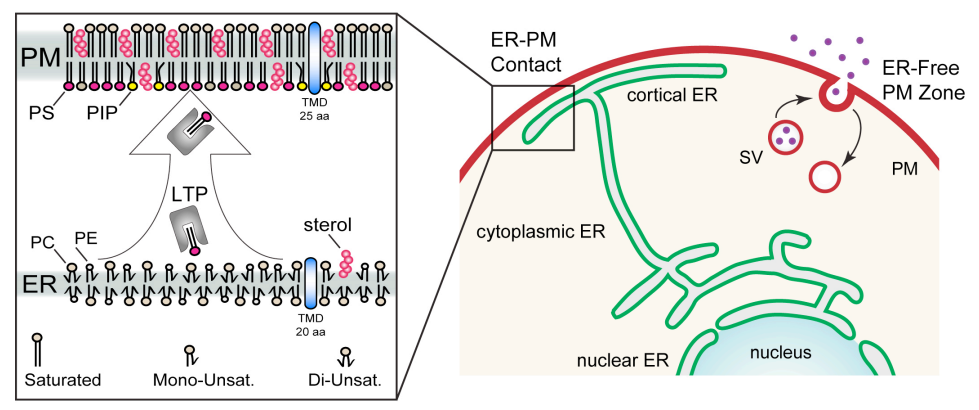

Figure 1. The plasma membrane is organized into ER-associated domains, termed ER-PM contacts, and ER-free domains. At ER-PM contacts, close apposition of the cortical ER to the PM (spanning 10 to $30 \mathrm{~nm}$ apart) facilitates non-vesicular lipid exchange by specific lipid transfer proteins. The selective delivery of newly synthesized lipids from the ER to the PM by lipid transfer proteins establishes the unique lipid composition of the PM including high sterol lipid content and phosphatidylserine species with long saturated acyl chains. This endows the PM with its unique chemical and physical properties including high negative charge, high membrane lipid packing order, and a bilayer thickness to accommodate PM proteins with long transmembrane domains. In contrast, the ER has a very different membrane lipid identity with low sterol levels and phospholipids with short unsaturated acyl chains resulting in decreased membrane lipid packing order and bilayer thickness. Vesicular trafficking events occur in ER-free PM domains. For this reason, ER-PM contacts and ER-free PM zones are often considered to be spatially and functionally distinct (i.e. sites for non-vesicular transport versus vesicular trafficking, respectively). However, it is becoming more and more clear that membrane lipid dynamics taking place at ER-PM contacts generate lipid and calcium signals necessary to sustain vesicle trafficking in ER-free zones. Mono-Unsat., mono-unsaturated lipid species; Di-Unsat., diunsaturated lipid species; LTP, lipid transfer protein; PC, phosphatidylcholine; PE, phosphatidylethanolamine; PIP, phosphatidylinositol phosphate; PS, phosphatidylserine; SV, secretory vesicle; TMD, transmembrane domain. 


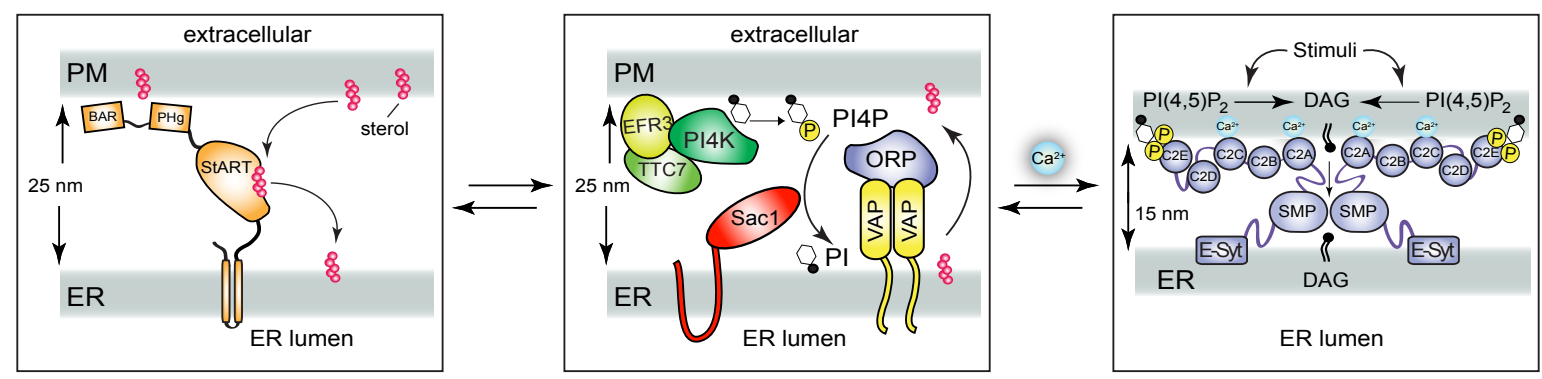

Figure 2. ER-PM contacts are assembled as needed by the specific recruitment of distinct lipid transfer proteins. (Center) The ORP (oxysterol-binding related proteins) lipid transfer proteins control plasma membrane lipid composition, organization, and identity. Certain ORP family members (including the yeast Osh2 protein) bind the ER-localized VAP proteins to form ER-PM contacts spanning approximately 25 to $30 \mathrm{~nm}$ in distance. ORP isoforms bind newly synthesized sterol lipids (e.g. ergosterol or cholesterol) at the ER and deliver the bound sterol lipid to the PM. The ORP lipid transfer proteins then bind PI4P generated by a phosphatidylinositol 4-kinase (PI4K) complex at the PM to prevent removal of sterol molecules enriched at the PM. The bound PI4P is hydrolyzed by the ER-localized Sac1 phosphatase for continued rounds of sterol delivery to the PM. In addition to sterols, the PM is highly enriched in negatively charged phosphatidylserine. ORP isoforms (the Osh6 and Osh7 proteins in yeast and the ER-localized ORP5 and ORP8 human orthologs) function at ERPM contacts as PS/PI4P exchange proteins to deliver newly synthesized PS from the ER to the PM (not shown; see main text for additional details). (Left) Conserved StARkin protein family members function as sterol transfer proteins at ER-PM contacts. PM organization and homeostasis not only requires delivery of sterol lipids to the PM but also the selective removal of sterol molecules from the PM. In yeast, StARkin family members bind sterol species (e.g. dihydroergosterol) using their StARTlike (StAR-related lipid-transfer) domains for retrograde transport of sterol from the PM to the ER. The StARkin family member Ysp1 is represented. Importantly, ER-PM contacts formed by the StARkin proteins are spatially distinct from ER-PM contacts where ORP family members function. In this manner, the StARkin and ORP lipid transfer protein activities may precisely balance sterol lipid levels, species composition, and distribution in the PM as needed. (Right) The extended synaptotagmins (ESyts) function as inducible lipid transfer proteins at ER-PM contacts. E-Syt proteins use their SMP (synaptotagmin-like and mitochondrial lipid-binding protein) domains to dimerize and transfer glycerolipids and glycerophospholipids down a concentration gradient (from high to low concentrations). Consequently, E-Syt proteins could potentially undue the phosphatidylserine gradient set up by ORP isoforms at ER-PM contacts. Accordingly, E-Syts proteins require close membrane tethering to function as lipid transfer proteins and may not be active at the 25 to $30 \mathrm{~nm}$ contacts formed by ORP-VAP assemblies (center panel). Moreover, E-Syts are tightly regulated and specifically activated upon receptor signaling coupled to phospholipase $\mathrm{C}$ activity and calcium $\left(\mathrm{Ca}^{2+}\right)$ influx. $\mathrm{Ca}^{2+}$ binding to the $\mathrm{C} 2 \mathrm{~A}$ and $\mathrm{C} 2 \mathrm{C}$ domains as well as $\mathrm{PI}(4,5) \mathrm{P}_{2}$ binding to the $\mathrm{C} 2 \mathrm{E}$ domain of the $\mathrm{E}-\mathrm{Syt} 1$ protein results in the formation of specialized close ER-PM contacts for the retrograde recycling of phospholipase C-generated diacylglycerol (DAG) from the PM to the ER. In this manner, the E-Syts may specifically participate in lipid and $\mathrm{Ca}^{2+}$ signaling events at the PM. 

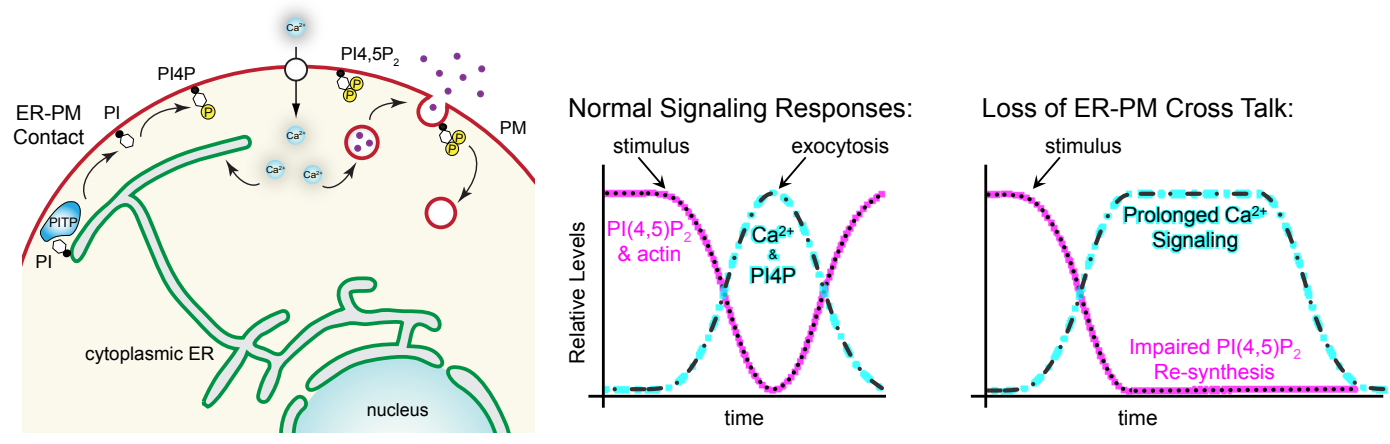

Figure 3. ER-PM contacts modulate phosphoinositide lipid and $\mathrm{Ca}^{2+}$ oscillations during regulated exocytosis. (Left) $\mathrm{Ca}^{2+}$ influx by the store-operated $\mathrm{Ca}^{2+}$-entry (SOCE) pathway and voltage-gated $\mathrm{Ca}^{2+}$ channels triggers the release of secretory cargo and the formation of specialized ER-PM contacts. This coordinated regulatory mechanism controls phosphoinositide lipid and $\mathrm{Ca}^{2+}$ dynamics necessary to sustain exocytic events at the PM. (Center) Stimuli that activate phospholipase $C$ and $\mathrm{PI}(4,5) \mathrm{P}_{2}$ hydrolysis subsequently result in $\mathrm{Ca}^{2+}$ influx (by SOCE) and increase PI4P levels necessary for $\mathrm{PI}(4,5) \mathrm{P}_{2}$ re-synthesis. The Nir2 and $\mathrm{Ca}^{2+}$-regulated TMEM24 phosphatidylinositol transfer proteins (PITP, left panel) facilitate PI4P synthesis at the PM following phospholipase C activation. In turn, PI4P activates the SOCE pathway and serves as a substrate for $\mathrm{PI}(4,5) \mathrm{P}_{2}$ re-synthesis. Importantly, these dynamic fluctuations in phosphoinositide lipid and $\mathrm{Ca}^{2+}$ signals, even while seemingly slight (less than two-fold changes), modulate the timing and extent of exocytic events at the PM. (Right) Transient disruptions in the homeostatic regulation of phosphoinositide and $\mathrm{Ca}^{2+}$ signaling at ER-PM contacts results in prolonged $\mathrm{PI} 4 \mathrm{P}$-regulated $\mathrm{Ca}^{2+}$ influx and impaired $\mathrm{PI}(4,5) \mathrm{P}_{2}$ re-synthesis. Long-term interruptions in ER-PM cross talk eventually lead to significant depletion of $\mathrm{PI}(4,5) \mathrm{P}_{2}$ pools and failure to generate cytosolic $\mathrm{Ca}^{2+}$ signals, a system-wide collapse of the $\mathrm{PI}$ kinase- $\mathrm{Ca}^{2+}$ signaling paradigm. Consequently, ER-PM contacts impact vital physiological processes including neurotransmission, insulin secretion, and immune cell signaling. 